\title{
NIGHT SLEEP ELECTROENCEPHALOGRAM POWER SPECTRAL ANALYSIS IN EXCESSIVE DAYTIME SLEEPINESS DISORDERS
}

\author{
RUBENS REIMÃO *
}

SUMMARY - A group of 53 patients (40 míales, 13 females) with mean age of 49 years, ranging from 30 to 70 years, was evaluated in the. following excessive daytime sleepiness (EDS) disorders: obstructive sleep apnea syndrome (B4a), periodic movements in sleep (B5a), affective disorder (B2a), functional psychiatric non affective disorder (B2b). We considered all adult patients referred to the Center sequentially with no other distinctions but these three criteria: (a) EDS was the main complaint; (b) right handed; (c) not using psychotropic drugs for two weeks prior to the all-night polysomnography. EEG (C3/A1, C4/A2) samples from 2 to 10 minutes of each stage of the first REM cycle were chosen. The data was recorded simultaneously in magnetic tape and then fed into a computer for power spectral analysis. The percentage of power (PP) in each band calculated in relation to the total EEG power was determined of subsequent sections of $20.4 \mathrm{~s}$ for the following frequency bands: delta, theta, alpha and beta. The PP in all EOS patients sample had a tendency to decrease progressively from the slowest to the fastest frequency bands, in every sleep stage. PP distribution in the delta range increased progressively from stage 1 to stage 4; stage REM levels were close to stage 2 levels. In an EDS patients interhemispheric coherence was high in every band and sleep stage. B4a patients sample PP had a tendency to decrease progressively

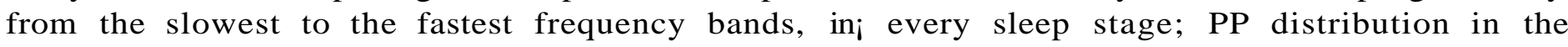
delta range increased progressively from stage 1 to stage 4; stage REM levels were between stage 1 and stage 2 levels. B2a patients sample PP had a tendency to decrease progressively from the slowest to the fastest frequency bands, in every sleep stage; PP distribution in the delta range increased progressively from stage 1 to stage 4; stage REM levels were close to stage 2 levels. B2b patients sample PP had a tendency to decrease progressively from the slowest to the fastest frequency bands, in every sleep stage; PP distribution in the delta range increased progressively from stage 1 to stage 3 ; stage 4 levels were close to stage 3 levels; stage REM levels were close to stage 2. B5a patients sample PP had a tendency to decrease progressively from the slowest to the fastest frequency bands, in every sleep stage; PP distribution in the delta range increased progressively from stage 1 to stage 3; stage REM levels were close to stage 2 levels, Interhemispheric coherences of B4a, B2b, and B5a groups were high in, every band and sleep stage. B4a, B2a, B2b, and B5a power spectral analysis comparison showed higher PP in B2b stage 1 alpha band, as well as, higher PP in B5a stage 2 theta band. The B4a versus. B2a power spectral analysis comparison showed higher PP in B4a stages 1 and REM alpha bands, as well as higher PP in B2a stage REM delta band.

Análise do espectro de potência do eletroencefalograma durante o sono: estudo de pacientes com sonolência excessiva diurna.

RESUMO - Foram avaliados 53 pacientes (média de idade - 49,0 anos; variação de 30-70 anos) com o objetivo de analisar o espectro de potencia do EEG durante o sono nos seguintes distúrbios que provocam sonolência excessiva diurna: síndrome de apnéia do sono tipo obstrutivo, movimentos periódicos do sono, distúrbios afetivos, distúrbios psiquiátricos funcionais não afetivos. Os dados obtidos indicam primeiramente que os diversos estágios do sono po-

* Sleep Disorders Center, Baptist Memorial Hospital, Memphis, Tennessee, USA. This research was partially supported by Coordenadoria de Aperfeiçoamento de Pessoal de Ensino Superior (CAPES).

Dr. Rubens Reimão - Divisão de Neurologia, Hospital das Clínicas, Faculdade de Medicina, Universidade de São Paulo - Caixa Postal 8091 - 01060 São Paulo SP - Brasil 
dem ser distinguidos quantitativamente entre os pacientes com sonolência excessiva diurna. Evidenciam, em segundo lugar, que os grupos diagnósticos têm padrões próprios de distribuição do espectro de potência. Em terceiro, mostram a ausência de assimetrias mantidas.

The all-night polysomnogram study is usually made by visual inspection, following standardized parameters 16. This is an accurate tool for clinical purposes. However, the computerized study of the electroencephalogram (EEG) during sleep revealed new aspects and details not visually detectable. The power spectral analysis was the first system utilized for quantitative EEG research during sleep, by Grass $\&$ col.8 in 1938, followed by Knott \& col.10 in 1942. The power spectrum (also called auto-spectrum of variance) shows the distribution of average intensity (mean square value or variance) of the EEG in relation to its frequency 5 . It may be studied in its original unit $\left(i \dot{i} V^{2}\right)$ but, more often, due to its methodological and statistical advantages ${ }^{14}$, it is applied as the percentage of power (PP) in a given frequency in relation to the power of a given spectrum. Sleep discorders accompanied by excessive daytime sleepiness (EDS) form the main group of patients who look for Sleep Disorders Centers, comprising 36.0 ot $42.2 \%$ of those $e^{2}$. In this respect, it is remarkable the lack of literature in connection with the use of this quantitative tool (the EEG power spectral analysis) in such pathological group.

The objective of this paper was to determine the EEG power spectrum during sleep in the following EDS disorders: sleep apnea syndrome (B4a), periodic movements in sleep (B5a), affective disorders (B2a), functional psychiatric non affective disorders (B 2b). This nomenclature and codes follow the international classification i.

\section{METHODS}

We evaluated 53 consecutive patients $(40$ men and 13 women) with EDS (Table 1). Mean age was 49.0 years (range 30 to 70 years). The selection criteria systematically applied were: (a) EDS was the main complaint; (b) right handed; (c) not using psychotropic drugs for two weeks prior to the polysomnography.

All-night standard polysomnography recordings included EEG (C3/A1; C4/A2) according to 10-20 System 9 criteria; electrooculogram using common reference (L.E/FP; FP/RE); electromyogram of submentalis and anterior tibialis muscles; buccal and nasal airflow measured by thermocouples; respiratory effort detected by thoracic and abdominal pneumograms; continuous transcutaneous oxygen saturation monitoring. EEG sensitivity of $7.5 \wedge \mathrm{V} / \mathrm{mm}$, high frequency filter of $90.0 \mathrm{~Hz}$, and low frequency filter of $1.0 \mathrm{~Hz}$ were used.

EEG samples of each sleep stage of the first REM cycle were obtained. Sample duration varied from 2 to $10 \mathrm{~min}$. An effort was made to obtain the longest possible time for each stage without the interference of artifacts, however, we established a maximum limit of 10 min. Slesep staging followed the standardized criteria of Rechtschaffen, \& col. 16. Data was simultaneously tape recorded, and liater fed into a computer (Med-80, Nicolet Inst. Co.), using a power spectral analysis software 15. Each BEG frequency band PP was calculated 19 in relation ot the total power for $20.4 \mathrm{~s}$ consecutive) segments, for the following bands: delta (0-3 Hz), theta (4-7 Hz), alpha $(8-12 \mathrm{~Hz})$ land beta $(13-20 \mathrm{~Hz})$.

The analyzed samples did not include awakenings, arousals, artifacts, apneas nor periodic leg movements. Such a selection was obtained employing three distinct quality controls: (1) during the visual scoring on the paper EEG records; (2) during visual scoring of the computer screen; (3) by means of computer filters for excessively high amplitude waves 11.

The evaluation of obtained data was performed in five steps: (a). The power distribution for the total sample of 51 patients with EDS, with meani values and a standard deviation (SD) were determined (Table 2), the interhemispheric differences for this group was calculated (Table 3). (b). The total sample was separated according to diagnostic categories; As B4a, B2a, B2b and B5a were the most frequent diagnosis, only they were taken for further analysis in steps (b), (c), and (d); mean PP, and SD were determined for each diagnostic group, in each stage and frequency band; interhemispheric differences were calculated for each diagnostic group, stage, and frequency band. (c). In order to determine PP distribution differences between sleep stages, for each band and each diagnostic group, Analysis of Variance test was applied (Tablet 4); furthermore, whenever the variance allowed 


\begin{tabular}{|c|c|c|c|c|c|c|c|c|c|c|c|}
\hline Case & Name & Age & Sex & Diagnosis & Diagnostic code & Case & Name & Age & Sex & Diagnosis & Diagnostic cod $\epsilon$ \\
\hline 1 & DD & 47 & $\mathbf{M}$ & OSAS & B4a & 27 & $\mathrm{JV}$ & 45 & $\mathbf{F}$ & $\mathrm{AD}$ & $\mathbf{B} \dot{\mathrm{s} a} \mathrm{a}$ \\
\hline 2 & LB & 38 & $\mathbf{M}$ & OSAS & B4a & 28 & $\mathrm{LC}$ & 66 & $\boldsymbol{F}$ & $\mathbf{A D}$ & B2a \\
\hline 3 & WM & 45 & $\mathbf{M}$ & OSAS & B4a & 29 & BW & 58 & $\mathbf{F}$ & OSAS & B4a \\
\hline 4 & $\mathbf{P W}$ & 41 & $\mathbf{M}$ & OSAS & B4a & 30 & GN & 59 & M & OSAS & B4a \\
\hline $\mathbf{5}$ & JL & 53 & $\mathbf{M}$ & Narcolepsy & B6 & 31 & LR & 39 & $\mathbf{M}$ & OSAS & B4a \\
\hline 6 & PR & 55 & $\mathbf{M}$ & OSAS & B4a & 32 & GF & 37 & $\mathbf{M}$ & PMS & B5a \\
\hline 7 & $\mathbf{F H}$ & 40 & $\mathbf{M}$ & OSAS & B4a & 33 & OJ & 54 & M & PMS & B5a \\
\hline 8 & HB & 65 & $\mathbf{M}$ & OSAS & B4a & 34 & $\mathrm{RE}$ & 34 & M & FPNAD & B2b \\
\hline 9 & LN & $\mathbf{5 5}$ & $\mathbf{M}$ & FPNAD & B2b & 35 & $\mathrm{JP}$ & 48 & $\mathbf{M}$ & NOF & B9b \\
\hline 10 & MR & 42 & $\mathbf{F}$ & $\mathrm{AD}$ & B2a & 36 & LG & 41 & M & OSAS & B4a \\
\hline 11 & JW & 64 & $\mathbf{M}$ & OSAS & $B 4 a$ & 37 & $\mathrm{~TB}$ & 70 & $\mathbf{M}$ & PMS & B5a \\
\hline 12 & $\mathbf{J M}$ & 50 & $\mathbf{M}$ & FPNAD & $\mathrm{B} 2 \mathrm{~b}$ & 38 & $\mathrm{JW}$ & 43 & $\mathbf{M}$ & FPNAD & B2b \\
\hline 13 & $J F$ & 37 & $\mathbf{F}$ & $A D$ & $\mathrm{~B} 2 \mathrm{a}$ & 39 & $\mathrm{DC}$ & 32 & F & $\mathrm{AD}$ & B2a \\
\hline 14 & $\mathrm{BD}$ & 47 & M & OSAS & B4a & 40 & $\mathrm{KM}$ & 68 & $\mathbf{M}$ & OSAS & B4a \\
\hline 15 & GD & 51 & $\mathbf{F}$ & $\mathrm{AD}$ & B2a & 41 & LG & 59 & $\mathrm{~F}$ & PMS & $\mathbf{B 5} \mathfrak{a}$ \\
\hline 16 & $R B$ & 40 & $\mathbf{M}$ & OSAS & B4a & 42 & $\mathrm{WJ}$ & 57 & $\mathbf{M}$ & OSAS & B4a \\
\hline 17 & $\mathrm{JR}$ & 34 & $F$ & $\mathrm{AD}$ & B2a & 43 & $\mathrm{JT}$ & 47 & Mi & $A D$ & B2a \\
\hline 18 & $\mathrm{CK}$ & 44 & $\mathbf{M}$ & OSAS & $\mathrm{B} 4 \mathrm{a}$ & 44 & $J G$ & 59 & $\mathbf{M}$ & OSAS & B4a \\
\hline H & JP & 52 & $\mathbf{M}$ & OSAS & B4a & 45 & $\mathrm{GE}$ & 51 & M & OSAS & B4a \\
\hline 20 & EH & 65 & $\mathbf{M}$ & Narcolepsy & $\mathrm{B6}$ & 46 & $\mathrm{CC}$ & 48 & $\mathrm{M}$ & OSAS & B4a \\
\hline 24 & RG & 49 & $\mathbf{M}$ & OSAS & B4a & 47 & WP & 39 & $\mathbf{M}$ & FPNAD & $\mathrm{B} 2 \mathrm{~b}$ \\
\hline 22 & $A B$ & & $\mathrm{~F}$ & ADAD & B4a & 48 & VR & 68 & $\mathbf{F}$ & OSAS & B4a \\
\hline $\begin{array}{l}22 \\
22\end{array}$ & $\mathrm{AB}$ & 42 & F & AD & B2a & 49 & SL & 50 & $\mathbf{M}$ & OSAS & B4a \\
\hline 23 & $\mathbf{P G}$ & 35 & M & OSAS & B4a & 50 & BS & $\mathbf{5 5}$ & $\mathbf{M}$ & $\mathbf{A D}$ & B2a \\
\hline 24 & $\mathrm{DV}$ & 48 & F & PMS & B5a & 51 & JD & 64 & M & OSAS & B4a \\
\hline 25 & $\mathrm{DC}$ & 43 & $\mathbf{F}$ & NOF & $\mathrm{B} 10 \mathrm{~b}$ & 52 & JB & 61 & $\mathbf{M}$ & OSAS & B4a \\
\hline 26 & MH & 30 & $\mathbf{M}$ & $\mathrm{AD}$ & B2a & 53 & WJ & 33 & $\mathbf{M}$ & OSAS & B4a \\
\hline
\end{tabular}

Table 1 - Identification data of 53 patietns with excessive daytime sleepiness.

$M$, male; F, female; age in years; FPNAD, functional psychiatric non affective disorder; OSAS, obstructive sleep apnea syndrome; AD, affec. tive disorder; PMS, periodic movements in sleep; Diagnosis and Diagnostic code: see reference 1. 


\begin{tabular}{|c|c|c|c|c|c|c|}
\hline & & $\stackrel{1}{N=53}$ & $\stackrel{2}{N=} 51$ & $\begin{array}{c}3 \\
\mathbf{N}=33\end{array}$ & $\begin{array}{c}4 \\
N=9\end{array}$ & $\begin{array}{c}\text { REM } \\
\mathbf{N}=\mathbf{5 3}\end{array}$ \\
\hline \multirow[t]{2}{*}{ Delta } & $\mathbf{R}$ & $32.5 \pm 13.2$ & $50.8 \pm 12.3$ & $65.5 \pm 13.2$ & $70.1 \pm 8.9$ & $45.6 \pm 12.5$ \\
\hline & $\mathrm{L}$ & $35.0 \pm 13.6$ & $52.3 \pm 12.3$ & $66.9 \pm 14.0$ & $72.0 \pm 10.5$ & $47.2 \pm 12.1$ \\
\hline \multirow[t]{2}{*}{ Theta } & $\mathrm{R}$ & $22.9 \pm 6.9$ & $20.3 \pm 3.7$ & $16.4 \pm 4.2$ & $15.2 \pm 5.8$ & $24.7 \pm 6.2$ \\
\hline & $\mathbf{L}$ & $22.5 \pm 7.2$ & $19.8 \pm 4.0$ & $16.0 \pm 4.3$ & $14.4 \pm 6.4$ & $24.7 \pm 6.4$ \\
\hline \multirow[t]{2}{*}{ Alpha } & $\mathbf{R}$ & $22.8 \pm 10.2$ & $16.3 \pm 6.3$ & $11.8 \pm 6.5$ & $11.2 \pm 5.2$ & $16.2 \pm 6.8$ \\
\hline & $\mathbf{L}$ & $21.7 \pm 9.8$ & $16.1 \pm 6.9$ & $11.2 \pm 6.8$ & $10.0 \pm 4.4$ & $15.8 \pm 7.2$ \\
\hline \multirow[t]{2}{*}{ Beta } & $\mathbf{R}$ & $13.6 \pm 6.1$ & $8.9 \pm 4.5$ & $4.7 \pm 4.3$ & $2.5 \pm 1.4$ & $9.3 \pm 4.6$ \\
\hline & $L$ & $13.1 \pm 6.2$ & $8.5 \pm 4.6$ & $4.1 \pm 3.6$ & $2.3 \pm 1.8$ & $8.7 \pm 4.7$ \\
\hline
\end{tabular}

Table $2-E E G$ percentages of power (PP) means and standard deviation distribution according to sleep stages and frequency bands, for both hemispheres $(R, L)$, in 53 patients with excessive daytime sleepiness.

\begin{tabular}{lccccc}
\hline & $\begin{array}{c}1 \\
\mathrm{~N}=53\end{array}$ & $\begin{array}{c}2 \\
\mathrm{~N}=51\end{array}$ & $\mathrm{~N}=33$ & $\begin{array}{c}4 \\
\mathrm{~N}=9\end{array}$ & $\begin{array}{c}\text { REM } \\
\mathrm{N}=53\end{array}$ \\
\hline Delta & $-2.4 \pm 7.2$ & $-1.4 \pm 4.2$ & $-1.3 \pm 3.6$ & $-1.8 \pm 4.2$ & $-1.6 \pm 5.1$ \\
Theta & $0.3 \pm 4.8$ & $0.5 \pm 1.6$ & $0.3 \pm 1.4$ & $0.7 \pm 1.1$ & $0.5 \pm 2.0$ \\
Alpha & $1.0 \pm 3.6$ & $0.2 \pm 2.5$ & $0.5 \pm 1.8$ & $1.2 \pm 2.1$ & $0.3 \pm 2.6$ \\
Beta & $0.4 \pm 5.5$ & $0.4 \pm 2.0$ & $0.6 \pm 2.7$ & $0.1 \pm 1.1$ & $0.6 \pm 2.1$ \\
\hline
\end{tabular}

Table $3-E E G$ percentages of power (PP) means and standard deviation interhemispheric differences, according to sleep stages and frequency bands, in 53 patients with excessive daytime sleepiness.

Interhemispheric difference $=R P P-L P P$.

\begin{tabular}{|c|c|c|c|c|}
\hline Diagnostic & Band & $\mathrm{DF}$ & $\mathbf{F}$ & $\mathbf{p}$ \\
\hline \multirow[t]{4}{*}{ B4a } & Delta & F4, 95 & 20.7 & 0.0000 \\
\hline & Theta & F4, 95 & 8.5 & 0.0000 \\
\hline & Alpha & F4, 95 & 6.1 & 0.0002 \\
\hline & Beta & $F 4,95$ & 8.2 & 0.0000 \\
\hline \multirow[t]{4}{*}{ B2a } & Delta & F4, 38 & 9.3 & 0.0000 \\
\hline & Theta & F4, 38 & 5.5 & 0.0013 \\
\hline & Alpha & F4, 38 & 1.2 & 0.3046 \\
\hline & Beta & F4, 38 & 5.9 & 0.0008 \\
\hline \multirow[t]{4}{*}{$\mathrm{B} 2 \mathrm{~b}$} & Delta & $F 4,15$ & 9.9 & 0.0004 \\
\hline & Theta & $F 4,15$ & 2.1 & 0.1289 \\
\hline & Alpha & $\mathrm{F} 4,15$ & 4.8 & 0.0104 \\
\hline & Beta & $\mathbf{F 4}, 15$ & 4.0 & 0.0210 \\
\hline \multirow[t]{4}{*}{ B5a } & Delta & F4, 18 & 6.5 & 0.0020 \\
\hline & Theta & F4, 18 & 2.2 & 0.1015 \\
\hline & Alpha & $\mathrm{F} 4,18$ & 2.7 & 0.0596 \\
\hline & Beta & F4, 18 & 4.4 & 0.0117 \\
\hline
\end{tabular}

Table 4 - Compurison between sleep stages EEG percentages of power (PP), accordiny to frequency bands and diagnosis.

Analysis of variance was applied. DF, degrees of freedom; $F, F$ distribution of Snedecor. 
and statistically applicable, pairs of variables were compared using the Newman-Keules test; thé Analysis of Variance was only applied after considered adequate by homogeneity tests of Cochrans and Bartlett. (d). PP distribution differences between diagnostic groups were compared using the Analysis of Variance test, for each stage and band (Table 5); NewmanKeules test was also used when statistically adequate, (e). The test, for each stage and frequency band was also used to establish a detailed comparison between diagnostic groups; this test was only applicable to B4a and B2a groups; groups B2b and B5a did not have sufficient sample size to use the! test at its best.

\section{RESULTS}

Data obtained follows the siame five steps described above:

a. The PP distribution in, the whole 53 EDS patients sample, showed a progressive decrease of PP from the highest to the lowest frequency bands, in every sleep stage (Table 2). PP in delta band increased progressively from stage 1 to 4 , and the $R E M$ values were close to stage 2. PP in theta band decreased progressively from stiage 1 to 4 , and the values of stage REM were higher than stage 1. PP in alpha band decreased progressively from stage 1 to 4 , and the values of stage REM were close to stage 2 . PP in beta band decreased progressively from stage 1 to 4 , and the values of stiage REM were close to stage 2 . The SD varied from 1.4 to 14.0 with a tendency of the higher SD to occur in the lowest frequency bands. The PP interhemispheric differences varied from -2.4 to 1.2 , with a tendency of the higher differences to occur in the lowest frequency bands (Table 3 ).

b. PP distribution in each diagnostic group was determined in this second step. The B4a group showed PP distribution similar to the whole EDS patients sample, with a progressive increase from the highest to* the lowest frequency bands, in every stage. $P P$ in each frequency band also showed distribution similar to the whole EDS sample except for the REM values in delta band that were between stages 1 and 2 values. SD varied between 0.8 and 18.8, with a tendency of the higher SD to occur in the lowest frequency bands. The PP interhemispheric differences varied from -3.8 to 1.5 , With a tendency of the higher differences to occur in the lowest frequency bands.

The B2a group showed PP distribution similar to the whole EDS patients sample, with a progressive increase from the highest to the lowest frequency bands, in every stage, except for the values of stage 4 theta and alpha bands that were close together. PP in each frequency band also showed distribution similar to the whole EDS sample except for: (1) stages 3 and 4 delta band were) close together; (2) stages 1 and 2 , as well as, stages 3 and 4 alpha bands were close together; (3) stage REM alpha band was close to stages 3 and 4. SD varied between 0.5 and 13.2, with a tendency of the higher SD to occur in the lowest frequency bands. The PP interhemispheric differences varied from -7.4 to 3.9 , with a tendency of the higher differences to occur in the lowest frequency bands.

The B2b group showed PP distribution similar to the whole EDS patients sample, with a progressive increase from the highest to the lowest frequency bands, in every stage, except for the stage 1 delta and theta bands values that were lower than the alpha band values. $P P$ in each frequency band also showed distribution similar to the whole EDS sample except for: (1) stages 3 and 4 delta bands were close together; (2) stage 2 theta band was slightly higher and stages 1,3 , and 4 were close together; (3) stages 3 and 4 beta bands asi well as alpha bands were close together. SD varied between 0.4 and 17.4 with a tendency of the higher SD to occur in the lowest frequency bands. The PP interhemispheric differences varied from -2.5 to 4.2 , with a tendency of the higher differences to occur in the lowest frequency bands.

The B5a group showed distribution similar to the whole EDS patients sample, with a progressive increase from the highest to the lowest frequency bands', in every stage, except for the stage 1 theta band that had a tendency to be lower than the alpha band. PP in each frequency band also showed similar distribution to the whole EDS sample except for: (1) stages 3 and 4 delta bands werei close together, with a slight tendency to be lower in, stage 4; (2) stages 1 and 2 , as well as, stages 3 and 4 theta bands were close together; (3) stage 4 alpha band was slightly higher than stagei 3 ; (4) stages 1 and 2 , as well as, stages 3 and 4 beta bands were close together. SD varied between 1.3 and 16.6, with a tendency of the higher SD to occur in the lower frequency bands. The PP interhemispheric differences varied from -3.1 to 2.6, with a tendency of the higher differences to occur in the lowest frequency bands. 


\begin{tabular}{cllll}
\hline Stage & Band & DF & F & p \\
\hline \multirow{2}{*}{2} & Delta & F3, 45 & 0.9 & 0.4027 \\
& Theta & F3, 45 & 1.7 & 0.1709 \\
& Alpha & F3, 45 & 4.3 & 0.0091 \\
& Beta & F3, 45 & 0.7 & 0.5053 \\
& Delta & F3, 44 & 1.0 & 0.3639 \\
& Theta & F3, 44 & 3.4 & 0.0308 \\
& Alpha & F3, 44 & 0.3 & 0.8243 \\
& Beta & F3, 44 & 0.0 & 0.9724 \\
& Delta & F3, 27 & 0.4 & 0.7407 \\
& Theta & F3, 27 & 0.5 & 0.6649 \\
& Alpha & F3, 27 & 0.0 & 0.9946 \\
& Beta & F3, 27 & 1.0 & 0.3751 \\
& Delta & F3, 5 & 3.1 & 0.1226 \\
& Theta & F3, 5 & 0.4 & 0.7027 \\
& Alpha & F3, 5 & 1.7 & 0.2689 \\
& Beta & F3, 5 & 1.4 & 0.3288 \\
& Delta & F3, 45 & 1.9 & 0.1397 \\
& Theta & F3, 45 & 0.7 & 0.5308 \\
& Alpha & F3, 45 & 2.4 & 0.0799 \\
& Beta & F3, 45 & 1.3 & 0.2750 \\
\hline
\end{tabular}

Table 5 - Comparison between diagnostic groups EEG percentages of power (PP) according to frequency bands and sleep stages.

Analysis of variance was lapplied. DF', degrees of freedom; $F, F$ distribution of Snedecor.

c. PP was compared between, the sleep stages, for every frequency band, according to the diagnostic groups (Table 4), showing significant differences in every comparison, except for: (1) B2a alpha band; (2) B2b theta band; (3) B5a theta and alpha bands.

Submitting only the B4a group to Newman-Keules test, based on all 4 frequency bands, all the sleep stages were significantly different from each other, except for the distinction beween stages 3 and 4 .

Submitting only the B2a group to Newmian-Keules test, based on all 4 frequency bands, all the sleep stages were significantly different from each other, except for the distinctions between stages 2 and 4 , as well as, stages 3 and 4 . There were no significantly different $P P$ pairs in the alpha band, at the $p<[0.05$ level.

Submitting only the B2b group to Newman-Keules test, based on all 4 frequency bands, only stage 1 could be differentiated from the others. There were no significantly different $P P$ pairs in the theta band, at the $p<0.05$ level.

Submitting only the B5a group to Newman-Keules test, based on all 4 frequency bands, all the sleep stages were significantly different from the others, except for the distinctions between the following pairs of stages: 1-2, 1-REM, 2-REM, 3-4, 2-4 and 4-REM, in the delta band; 1-2, 1-£, 1-REM, 3-4, 3-REM land 4-REM, in the beta band. There were no significantly different $P P$ pairs in theta and alpha bands, at the p<0.05 level.

d. PP in every, band and stage was compared between the four diagnostic groups (Table 5). They showed significant differences restricted to stage 1 alpha and stage 2 theta bands; When taking exclusively stage 1 alpha band -and comparing the diagnostic groups, it became evident that the significant difference was between B2a and B2b. When taking exclusively stage 2 theta band and comparing the diagnostic groups, it became evident that the significant difference was between B5a and all the others.

e. B4a PP was compared with B2a for each stage and frequency band. There were significant differences between these diagnostic groups in stage 1 alpha band, as well as, stage REM alpha and delta bands. In these; differences, the B4a diagnostic group had higher alpha band values; the B2a diagnostic group had higher delta band values. 
The power spectral analysis methodology is particularly adequate for studying sleep due to its capacity of detecting a consistent oscilation, phasic or tonic, in a limited time period, even if this activity has small amplitude ${ }^{12}$. Gasser \& col. ${ }^{7}$ have demonstrated that the relative potency, such as we have used in this study, gives more reliable results than the use of absolute potency, which is subject to individual variations. Matouse ${ }^{14}$ also recommends to present it as $P P$ in each band, as it was done in this report. In this research, the EEG derivations followed the classical ones that have been used in sleep for the last two decades ${ }^{16}$. The option to register the spectrum potency without common reference was due to the smaller distortion of the potentials, as recommended by Fein \& $\operatorname{col} A$

The literature reveals a lack of studies with similar methodology, utilizing the power spectral analysis in EDS patients. The PP distribution pattern in our total EDS patients sample showed PP reduction from the lowest to the highest frequency bands, in all sleep stages. This pattern was previously described in normal adults 5.8.10. The same pattern was found in our subgroups, when divided according to the diagnosis.

Our total EDS sample showed increase of the $P P$ in delta band from stage 1 to 4; this band values in stage $R E M$ are similar to stage 2 . This increase was also previously described in normal adults 5. Such finding indirectly shows the sleep stages (particularly stages 2,3 and 4) definition criteria, which takes into consideration the proportion of high amplitude delta waves in a given time-limited sample 16.

In the total EDS sample, theta band had progressive decrease from stage 1 to 4, with REM values higher than stage 1 . In the alpha band, there was also a progressive reduction from stage 1 to 4 , with stage $R E M$ values similar to stage 2. In the beta band, there was a decrease from stage 1 to 4 , and the stage $R \mathbf{E}$ values were similar to stage 2 . The difficulty in distinguishing between stage REM and stage 1, by this method^, , s usually attributed to the similarity of their power spectral distributions. However, our data suggests that the differentiation between these two stages, or between stage REM and stage 2 should be considered in a dynamic perspective, as it changes according to the frequency band, as well as, the pathology taken into consideration. Stage REM is heterogeneous in its time course, e.g. showing sawtooth waves that are detected in the power spectral analysis as a PP increase in theta band. These irregularly distributed waves along REM sleep change the $P P$ in a given sample and so does the comparison with stages 1 and 2 .

The beta band peak in stage 2 , around 13-15 Hz, corresponding to sleep spindles 5, can not be detected by the method we have used as the potency is distributed for the whole beta band. The absence of peaks does not prevent the comparison between stages because the beta band potency will tend to elevate reflecting the spindles. It also should be considered that the increase in sigma band in non-REM sleep does not always correspond to spindles, and an increase in this band may occur even without visually detectable spindles $^{3} »^{4}$.

The progressive increase in delta band PP, from stage 1 to 4 allows a quantitative distinction between these stages and is partially related to the definition criteria that considers the percentage of delta contingent 16. However, the power spectral analysis determines the delta potency in a given time and not the duration of delta waves in this given sample. In spite of being distinct parameters, some previous data suggests that the power spectral analysis may be utilized for sleep staging with the usual visual standardization 16. The two methods show an agreement of $85-92 \%$ in normal adults records 17.

Tanguay \& col.20 described the EEG power spectrum in normal children. In the 34-56 months old, the 11-15 Hz band had PP of 3.3 in stage 2 and 1.8 in the same band in stage REM. Comparing with our results, beta band $P$ P are respectively 8.9 at right and 8.5 at left hemispheres, as well as 9.3 at right and 8.7 at left. Regarding the 0-3 Hz band, those children 20 had stage 2 PP of 66.5 and stage REM PP of 73.6. In our sample, delta band $P P$ was 50.8 at right and 52.3 at left in stage 2 ; 45.6 at right and 47.2 at left in stage REM. This comparison supports and objectively quantifies the well known decline of delta band from infancy to adulthood.

The interhemispheric coherence was elevated in our sample. This high coherence in every frequency band and sleep stages is in agreement with normal population data, from children to adults except for the first three months of life 3.4,6,18. 
Our power spectral analysis data of the four major EDS diagnostic groups points out that the sleep stages may be quantitatively distinguished by this method. Second, it shows that different diagnostic groups have distinct power spectral patterns. Third, it gives quantitative support to the lack of marked sustained asymetries concept, in every diagnostic group. Finally, it suggests that broader use of quantitative EEG power spectral analysis could help differentiating and understanding the EDS diseases.

Acknowledgements - Our gratitute to Helio Lemmi, M.D., Director of the Baptist Memorial Hospital Sleep Disorders Center for his advice and support. We thank also Aron Diament, M.D., Roger Vander Zwaag, Ph.D., Hagop Akiskal, M.D., Aldo Bevilacqua, M.D., Joseph Belluomini, Ronald Cowan and Sharon Burt for invaluable help, statistical, and technical assistance.

\section{REFERENCES}

1. Association of Sleep Disorders Centers. Classification of sleep and arousal disorders. Sleep 1979, 2:1.

2. Coleman RM. Diagnosis, treatment, and follow-up of about 8,000 sleep/wake disorder patients. In Guilleminault C, Lugaresi E (eds): Sleep/Wake Disorders: Natural History, Epidemiology, and Long-term Evolution. New York. Raven Press 1983, p 87-98.

3. Dumermuth G, Gasser T, Lange B. Aspects of EEG analysis in the frequency domain. In Dolce G, Kunkel $\mathrm{H}$ (eds): Computerized EEG Analysis. Stuttgart: Gustav Fisher Verlag, 1975, p 429-457.

4. Dumermuth G, Scollo-Lavizzari G. Spectral analysis of EEG in normal adults. In Koela WP, Levin P (eds): Sleep - Physiology, Biochemistry, Psychology, Pharmacology, Clinical Implications. Basel: Karger, 1973, p 246-249.

5. Dumermuth G, Waltz W, Scollo-Lavizzari G, Kleiner B. Spectral analysis of EEG activity in different sleep stages in normal adults. Eur Neurol 1972, 7:265.

6. Fein G, Raz J, Brown FF, Merrin EL. Common reference coherence dato, are counfounded by power and phase effects. Electroencephalogr Clin Neurophysiol 1988, 69:581.

7. Gasser T, Verleger R, Bacher P, Sroka L. Development of the EEG of school-age children and adolescents: I. Analysis of band power. Electroencephalogr Clin Neurophysiol 1988, 69:91.

8. Grass AM, Gibbs FA. A Fourier transform of the electroencephalogram. J Neurophysiol 1938, $1: 521$

9. Jasper HH. Report of the committee on methods of clinical examination in electroencephalography. Electroencephalogr Clin Neuropsysiol 1958, 10:371.

10. Knott JR, Gibbs A, Henry CE. Fourier transform of electroencephalogram during sleep. J Exper Psychol 1942, 31:465.

11. Ktonas PY, Osorio PL, Everett RL. Automated detection of EEG artifacts during sleep: preprocessing for all-night spectral analysis. Electroencephalogr Clin Neurophysiol $1979,46: 382$.

12. Kuwahara H, Higashi H, Mizuki Y, Matsunari S, Tanaka M, Inanaga K. Automatic real time analysis of human sleep stages by an interval histogram method. Electroencepha$\log \mathrm{r}$ Clin Neurophysiol 1988, 70:220.

13. Larsen LE, Walter DO. On automatic methods of sleep staging by EEG spectral power during REM periods. Sleep Res 1981, 10:151

14. Matousek M. Frequency analysis: processing of output data. In Remond A, (ed): Handbook of Electroencephalography and Clinical Neurophysiology. Amsterdam: Elsevier 197), 5A61-5А 66

15. Nicolet Instrument Corporation. Nicolet Med-80 Frequency Analysis Software. Wisconsin: Nicolet, 1975

16. Rechtschaffen A, Kales A. Manual of Standardized Terminology, Techniques and Scoring for Sleep Stages of Human Subjects. Los Angeles: Brain Information Service, University of California, 1968

17. Salinsky M, Goins S, Sutula T, Roscoe D, Wever S. Comparison of sleep staging by polygraph and color density spectral array. Sleep 1988, 11:131.

18. Schulte FJ, Bell B. Bioelectric brain development. Neuropediatrics 1973, 4:30.

19. Sciaretta G, Erculiani P. A proposal for calibrating EEG spectograms. Electroencephalogr Clin Neurophysiol 1978, $45: 674$.

20. Tanguay PE, Ornitz EM, Bozzo E, Doubleday C. EEG spectra in children during REM and stage 2 sleep. Sleep Res 1974, 3:81. 